\title{
Not all sellar masses are macroadenomas: think also in metastasis
}

\author{
Nem todas massas selares são macroadenomas: pense também em metástases \\ Paulo Victor Sgobbi de Souza ${ }^{1}$. Wladimir Bocca Vieira de Rezende Pinto ${ }^{2}$, Adrialdo José dos Santos ${ }^{2}$
}

A 54-year-old woman presented with a 3-month-history of blurred vision and refractory headache. Examination revealed severe bilateral visual acuity compromise, normal campimetry and bilateral papilledema. As a mean to investigate raised intracranial pressure, neuroimaging studies were performed and showed extensive sellar mass (Figure). Patient underwent transsphenoidal approach for resection of pituitary mass which disclosed metastasis of neuroendocrine lung tumor. Chest CT showed a mass in the anterior segment of right lower lobe of lung. Although symptomatic pituitary gland tumors are common in clinical practice ${ }^{1}$, metastatic disease represent a rare cause of sellar tumors, mainly of breast and lung primary origin ${ }^{2}$.
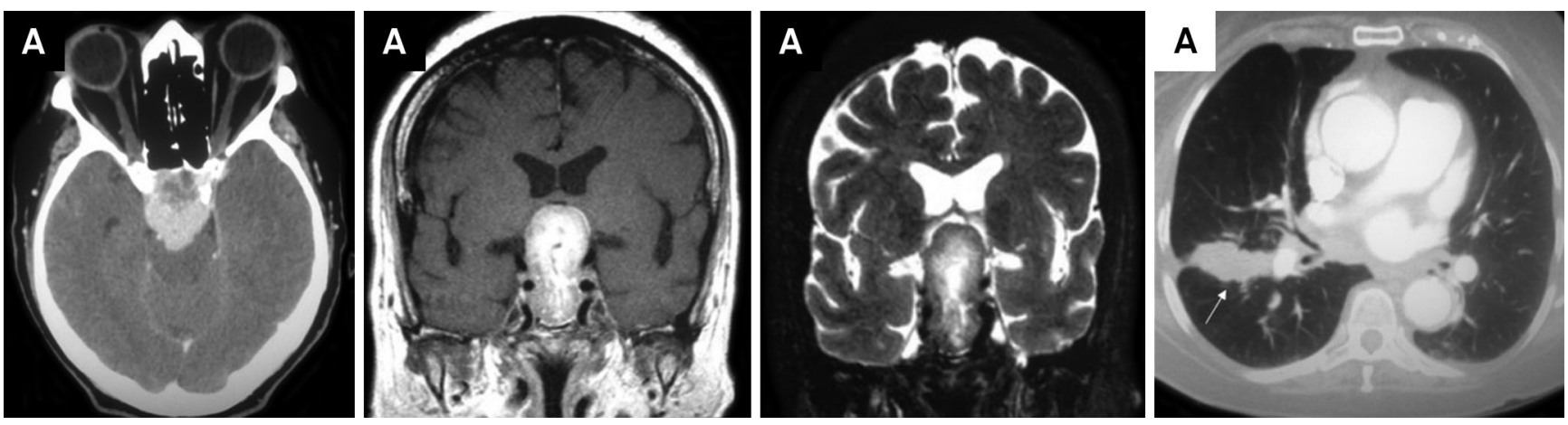

Figure. (A) Cranial CT scan showing a solid lesion in the sellar and suprasellar region with heterogeneous enhancement; (B) Coronal brain MRI sections disclosing a mass with heterogeneous enhancement on post-gadolinium T1 weighted images; (C) and with heterogeneous signal in T2-weighted images, hyperintense in its central portion and hypointense in its periphery; (D) Chest CT scan showing the presence of an irregular consolidation in the anterior basal segment of the lower lobe of the right lung associated with partial atelectasis (white arrow).

\section{References}

1. Rennert J, Doerfler A. Imaging of sellar and parasellar lesions. Clin Neurol 2. Neurosurg. 2007;109(2):111-24. http://10.106/j.clineuro.2006.11.001

Komninos J, Vlassopoulou V, Protopapa D, Korfias S, Kontogeorgos G, Sakas DE. Tumors metastatic to the pituitary gland: case report and literature review. J Clin Endocrinol Metab. 2004;89(2):574-80. http:// dx.doi.org/10.1210/jc.2003-030395

\footnotetext{
'Universidade Federal de São Paulo, Sao Paulo SP, Brazil;

${ }^{2}$ Departamento de Neurologia e Neurocirurgia, Universidade Federal de São Paulo, Sao Paulo SP, Brazil. Toledo, 650; 04023-900 São Paulo SP, Brasil; E-mail: wladimirbvrpinto@gmail.com

Conflict of interest: There is no conflict of interest to declare.

Received 20 May 2014; Received in final form 20 August 2014; Accepted 08 September 2014.
}

Correspondence: Wladimir Bocca Vieira de Rezende Pinto; Departamento de Neurologia e Neurocirurgia, Universidade Federal de São Paulo; Rua Pedro de 